\title{
Detektion von Flachstellen an Radreifen auf der Basis von Schallmessungen am Schienenkörper
}

\author{
Ralf Vandenhouten, Manfred Deutzer
}

\section{Einführung}

Von der Firma Deutzer Technische Kohle GmbH (DTK) wurde ein Messsystem zur Erfassung des Körperschalls an Schienen entwickelt, mit dem der Verschleiß an Radreifen von Schienenfahrzeugen ermittelt werden kann. Die Schallsignale werden im normalen Fahrbetrieb bei der Überfahrt des Schienenabschnitts, in dem sich der Vibrationssensor befindet, erfasst und als digitale Zeitreihen aufgezeichnet. Mit Hilfe eines Induktionssensors können die Zeitpunkte bestimmt werden, zu denen sich die Radreifen jeweils genau über dem Messpunkt in der Schiene befunden haben [1].

Im Falle von schadhaften Radreifen wird das normale Körperschallsignal überlagert von scharfen Impulsen hoher Intensität, die von den Stößen der Flachstellen auf der Schiene verursacht werden. Die Höhe eines solchen Impulses ist zum einen von der Ausprägung der Flachstelle abhängig, zum anderen aber auch von der Entfernung vom Sensor, in der der Impuls erzeugt wurde. Diese hängt von der Radstellung relativ zur Schienenposition ab und kann nicht beeinflusst werden, da im normalen Fahrbetrieb gemessen werden soll. In diesem Forschungsvorhaben wurde deshalb ein Analyseverfahren entwickelt, das die Flachstellenimpulse in geeigneter Weise normiert, so dass unabhängig von der Radstellung auf den Verschleiß einzelner Radreifen geschlossen werden kann.

\section{Theorie}

\subsection{Radreifen ohne Flachstellen}

Zunächst wird von einem unbeschädigten Radreifen ausgegangen. Ein solcher erzeugt aufgrund von Reibung und kleinen Unebenheiten eine Vibration bestimmter Intensität an der Stelle der Schiene, an der er aufliegt. Diese Vibration breitet sich als Körperschallwelle in der Schiene aus. Die Wellengeschwindigkeit in Stahl ist sehr hoch und kann für unsere Zwecke als unendlich angenommen werden. Die Welle wird jedoch von der Schiene und ihrer Einfassung gedämpft, wobei die Dämpfung als linear angenommen werden kann. Auf einem kleinen Gleisstück $d x$ erfährt die Intensität $I$ (bei Ausbreitung in positiver $\mathrm{x}$-Richtung) also eine Abschwächung $d I$, für die gilt:

$d I=-\lambda I d x$

wobei $\lambda$ die Dämpfungskonstante ist. Damit erhält man die lineare Differentialgleichung

$\frac{d I}{d x}=-\lambda I$
Bezeichnet man die Schallintensität an der Radposition $x_{0}$ mit $I_{0}$ und berücksichtigt die Tatsache, dass die Welle sich in negativer $\mathrm{x}$-Richtung symmetrisch ausbreitet, erhält man als Lösung der Differentialgleichung folgende Intensitätsfunktion:

$I(x)=I_{0} e^{-\lambda \mid x-x_{0}}$

Man beachte, dass der Dämpfungsfaktor $\lambda$ im Wesentlichen nur von der Beschaffenheit der Schiene und ihrer Einfassung abhängig ist, nicht hingegen vom Radreifen oder der Geschwindigkeit, mit der das Schienenfahrzeug fährt. Mit Hilfe der Formfunktion

$f_{\lambda}(x) \equiv e^{-\lambda \mid x}$

lässt sich die Intensitätsfunktion schreiben als

$I(x)=I_{0} f_{\lambda}\left(x-x_{0}\right)$

Bewegt sich das Schienenfahrzeug mit (zumindest annähernd) konstanter Geschwindigkeit v, ergibt sich für die am Sensor gemessene Intensitätszeitreihe

$I_{N}(t)=I_{0} f_{\lambda}\left(v \cdot\left(t-t_{0}\right)\right)=I_{0} f_{\lambda v}\left(t-t_{0}\right)$

wobei $t_{0}$ der Zeitpunkt ist, zu dem sich das Rad genau über dem Sensor befindet.

\subsection{Radreifen mit Flachstellen}

Eine Flachstelle an einem Radreifen erzeugt eine periodische Störintensität $I_{S}$, die beschrieben werden kann durch:

$I_{S}(t)=I_{1} \sum_{k=-\infty}^{\infty} \delta\left(t-k \Delta t-t_{d}\right) f_{\lambda_{k}}\left(t-t_{0}\right)$

wobei $\Delta t$ die Dauer einer Radumdrehung und $t_{d}$ den Zeitversatz zwischen Überfahren des Sensors und Auftreten des ersten Impulses bezeichnet. Die (diskrete) Dirac-Funktion $\delta$ ist dabei definiert als

$\delta(t)= \begin{cases}1 & \text { für } t=0 \\ 0 & \text { sonst }\end{cases}$

Der Faktor $f_{\lambda v}$ in Gleichung (7) ergibt sich wieder aus den Überlegungen zum Dämpfungsverhalten gemäß Abschnitt 2.1. Die Konstante $I_{1}$ ist ein Maß für den Verschleiß des Radreifens. Sie hängt allerdings auch von der Sensorkalibrierung sowie von der Geschwindigkeit ab, mit der sich das Schienenfahrzeug bewegt.

Die gesamte gemessene Schallintensität $I(t)$ eines Radreifens ergibt sich nun als Summe der Intensitäten $I_{N}$ 
und $I_{S}$ gemäß den Gleichungen (6) und (7) sowie einem konstanten Offset (Rauschpegel) $I_{R}$ :

$I(t)=I_{N}(t)+I_{S}(t)+I_{R}$

\subsection{Normiertes Maß für den Radreifenverschleiß}

Wie bereits erwähnt, ist der Wert von $I_{l}$ ein Maß für die Größe einer Flachstelle des Radreifens. Er ist allerdings nicht als Normmaß geeignet, da er in der Praxis von der Sensorkalibrierung und von der Geschwindigkeit des Schienenfahrzeugs abhängig ist. Der Zusammenhang zwischen Geschwindigkeit und Schallpegel wurde bereits in [1] untersucht. Dort wird ein exponentieller Anstieg der Intensität mit der Geschwindigkeit vermutet. Aus physikalischen Erwägungen erscheint ein quadratischer Anstieg eher wahrscheinlich.

In jedem Fall ist die Geschwindigkeitsabhängigkeit problematisch und sollte aus dem gesuchten Normmaß für den Verschleiß eliminiert werden. Glücklicherweise ist die normale (d.h. verschleißfreie) Intensitätsamplitude $I_{0}$ in gleicher Weise von der Kalibrierung und der Fahrzeuggeschwindigkeit abhängig. Wir definieren deshalb den normierten Verschleißgrad $\gamma$ eines Radreifens wie folgt:

$\gamma=\frac{I_{1}}{I_{0}}$

Aufgrund obiger Überlegungen ist $\gamma$ invariant gegenüber der Sensorkalibrierung und Änderungen der Geschwindigkeit. Je ausgeprägter eine Flachstelle ist, umso größer wird der Wert von $\gamma$. Für verschleißfreie Radreifen gilt $\gamma=0$. Damit ist $\gamma$ ein geeignetes Normmaß für den Grad des Radreifenverschleißes.

Es bleibt allerdings noch die Frage zu klären, wie diese theoretische Größe aus den Messungen bestimmt werden kann. Direkt gemessen werden kann nur die Intensität $I(t)$ gemäß (9) sowie der Offset $I_{R}$. Wenn zusätzlich auch die „verschleißfreie“ Intensität $I_{N}(t)$ bekannt ist, kann man folgende Zeitreihe (im Folgenden als normierte Verschleißkurve bezeichnet) berechnen:

$\Gamma(t) \equiv \frac{I(t)-I_{R}}{I_{N}(t)}-1=\frac{I_{N}(t)+I_{S}(t)}{I_{N}(t)}-1=\frac{I_{S}(t)}{I_{N}(t)}$

Mit Hilfe der Gleichungen (6) und (7) erhält man nun

$\Gamma(t)=\frac{I_{1} \sum_{k=-\infty}^{\infty} \delta\left(t-k \Delta t-t_{d}\right) f_{\lambda_{v}}\left(t-t_{0}\right)}{I_{0} f_{\lambda v}\left(t-t_{0}\right)}=\gamma \sum_{k=-\infty}^{\infty} \delta\left(t-k \Delta t-t_{d}\right)(12)$.

$\Gamma(t)$ ist also eine kammartige Funktion mit ausgeprägten Maxima, deren Höhe dem Verschleißgrad $\gamma$ entspricht. Dieser kann also aus $\Gamma$ unmittelbar abgelesen werden. Gleichung (11) beschreibt eine Transformation der instationären Signale $I_{S}(t)$ und $I_{N}(t)$ in die stationäre Zeitreihe (Näheres dazu siehe [2]).

\section{Ermittlung der Kurvenparameter}

Im Prinzip liefern die Gleichungen (11) und (12) die Vorgehensweise, wie der Verschleißgrad $\gamma$ bestimmt werden kann. Allerdings kommt darin auch die verschleißfreie Intensität $I_{N}(t)$ vor, die nicht bekannt ist und auch nicht direkt gemessen werden kann. Dieser Abschnitt behandelt die empirische Bestimmung der fehlenden Parameter.

\subsection{Rauschpegel}

Das gemessene Schallsignal $I(t)$ enthält den konstanten Rauschpegel $I_{R}$, der zunächst gemäß Gleichung (11) subtrahiert werden muss. Er kann leicht bestimmt werden, indem das Messsignal am Rand (z.B. lange bevor sich ein Schienenfahrzeug dem Sensor nähert) untersucht und dessen Mittelwert bestimmt wird. Dieser wird von allen Messwerten subtrahiert, so dass man ein Signal ohne Offset $\left(I_{R}=0\right)$ erhält.

\subsection{Glättung}

In der Praxis ist das gemessene Signal natürlich nicht so glatt, wie die Theorie es beschreibt, sondern weist mehr oder weniger starke Schwankungen auf. Um bei der Bestimmung der Kurvenparameter den Fehler zu minimieren, sollte im nächsten Schritt das Messsignal $I(t)$ mit Hilfe eines Tiefpassfilters geglättet werden. Gut geeignet ist dafür z.B. ein Gaußfilter, d.h. die Faltung des Signals mit einer Gaußkurve. Die Halbwertsbreite der Gaußkurve sollte ca. 20 Messwerte betragen, d.h. bei einer Abtastfrequenz von $100 \mathrm{~Hz}$ beispielsweise $200 \mathrm{~ms}$.

\subsection{Logarithmische Transformation}

Das geglättete Signal verschieben wir nun auf der Zeitachse so, dass der Sensor zum Zeitpunkt $t=0$ vom Radreifen überfahren wird (d.h. $t_{0}=0$ ). Von den beiden abfallenden Flanken des Signals wird nur diejenige weiterverarbeitet, die nicht in Richtung der anderen Achse desselben Drehgestells zeigt, d.h. bei der ersten Achse die linke Flanke und bei der zweiten Achse die rechte Flanke, so dass der betrachtete Signalabschnitt möglichst wenig Störungen anderer Räder enthält. Im Falle der linken Flanke wird das Signal außerdem noch an der y-Achse $(t=0)$ horizontal gespiegelt, so dass in jedem Fall ein bei $t=0$ beginnendes, abfallendes Signal das Resultat ist, das man nach einem angemessenen Zeitintervall (vor Erreichen des Rauschpegels, d.h. je nach Verlauf ca. bei $t=1-4 \mathrm{~s}$ ) abschneidet. Die Wertepaare $\left(t_{i}, y_{i}\right)$ dieser Zeitreihe gehorchen nun, lässt man die einzelnen Störimpulse durch Flachstellen außer Acht, gemäß (6) näherungsweise der Beziehung

$y_{i}=I_{0} f_{\lambda v}\left(t_{i}\right)=I_{0} e^{-\lambda v t_{i}}$

Definiert man für alle positiven $y_{i}$ (die anderen werden nicht weiterverwendet)

$z_{i} \equiv \log y_{i}$

so gilt für alle $i$ :

$z_{i}=\log I_{0}-\lambda v t_{i}=a+b t_{i}$

d.h. die Wertepaare $\left(t_{i}, z_{i}\right)$ liegen im Idealfall auf einer Geraden mit Steigung $b=-\lambda v$ und Ordinatenabschnitt $a=\log I_{0}$. 


\subsection{Lineare Regression}

Die Gerade mit der geringsten quadratischen Abweichung kann durch lineare Regression der $N$ Wertepaare $\left(t_{i}, z_{i}\right)$ bestimmt werden. Man erhält für die Koeffizienten $a$ und $b$ in (15) dann

$$
\begin{aligned}
& a=\frac{\sum t_{i}^{2} \sum z_{i}-\sum t_{i} \sum t_{i} z_{i}}{N \sum t_{i}^{2}-\left(\sum t_{i}\right)^{2}} \\
& b=\frac{N \sum t_{i} z_{i}-\sum t_{i} \sum z_{i}}{N \sum t_{i}^{2}-\left(\sum t_{i}\right)^{2}}
\end{aligned}
$$

Die gesuchten Parameter erhält man daraus nun wie folgt:

$$
I_{0}=e^{a} \text { und } \lambda v=-b
$$

Diese kann man nun in (6) einsetzen und anschließend mit Hilfe von (11) und (12) den Verschleißgrad $\gamma$ bestimmen.

\section{Algorithmus}

Die in Abschnitt 3 detailliert beschriebene Vorgehensweise zur Bestimmung des Verschleißgrades lässt sich als Algorithmus in der Macrosprache der Signalverarbeitungssoftware Dataplore ${ }^{\circledR}[5]$ wie folgt umsetzen:

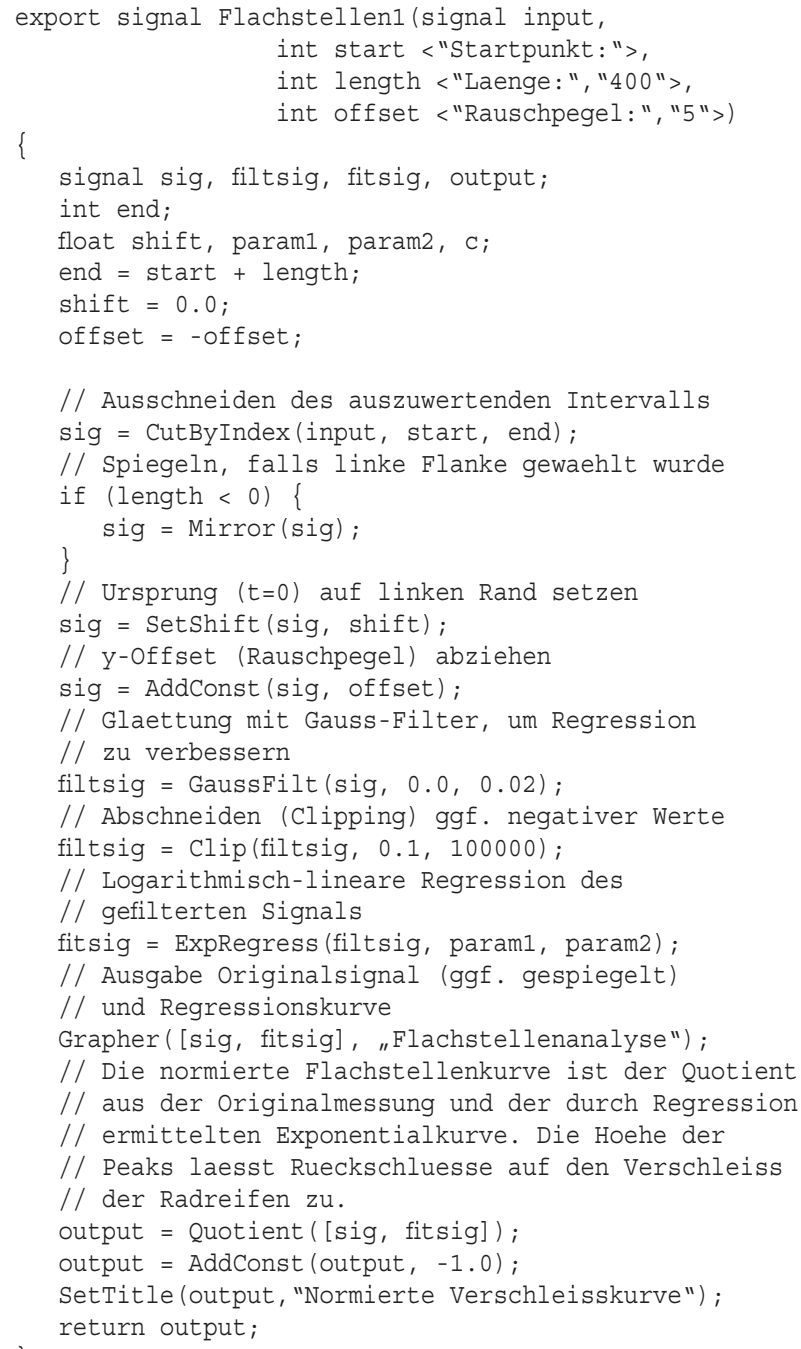

Die dabei ausgegebene normierte Verschleißkurve entspricht der Zeitreihe aus Gleichung (11) und (12). Die Höhe der Maxima dieser Zeitreihe gibt Aufschluss über den Verschleißgrad $\gamma$.

Ab welchem Wert von $\gamma$ eine Wartung bzw. ein Radwechsel vorzunehmen ist, kann anhand der zur Verfügung stehenden Beispieldaten nicht entschieden werden. Zur Festlegung eines solchen Grenzwertes sind entsprechende Referenzmessungen mit Radreifen, deren Verschleißzustand (z.B. aus einer optischen Vermessung) bekannt ist, erforderlich. Die bisherigen Analysen deuten jedoch darauf hin, dass für $\gamma<3$ von unkritischem Verschleiß ausgegangen werden kann, während $\gamma$-Werte über 5 auf zu hohen Verschleiß hindeuten.

\section{Ergebnisse}

Das vorgestellte Verfahren wurde an zahlreichen der zur Verfügung stehenden Datensätze getestet. Exemplarisch sollen hier einige Ergebnisse vorgestellt werden.

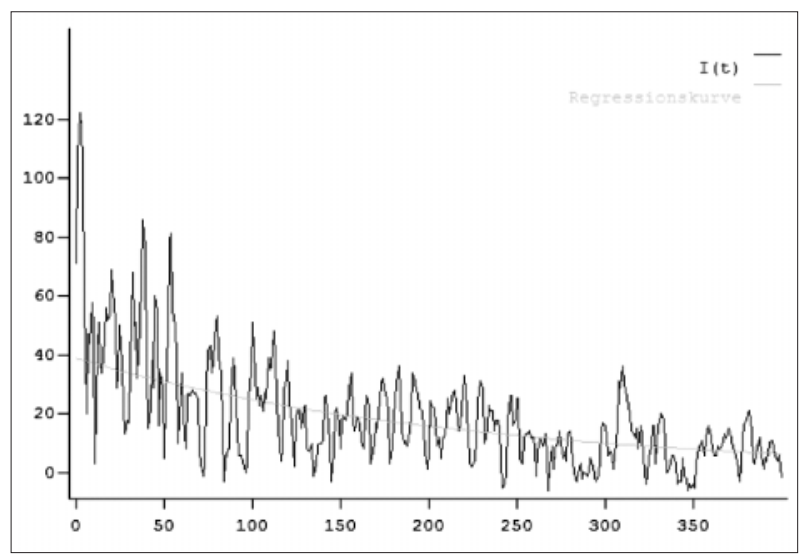

Abbildung 1a

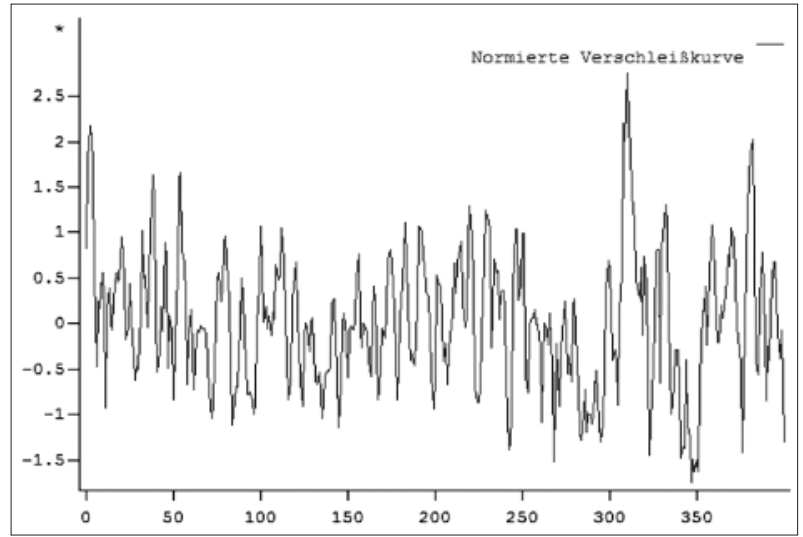

Abbildung $1 b$

Abbildung 1a zeigt die abklingende Intensitätskurve $I(t)$ eines intakten Radreifens aus einer Messung (Datensatz Vorführung/6213/2004-05-13-15-02-20) und die gemäß Abschnitt 3 ermittelte Regressionskurve für $I_{N}(t)$. Damit kann gemäß (11) die normierte Verschleißkurve $\Gamma(t)$ bestimmt werden, die in Abb. 1b zu sehen ist. Das Maximum, das hier bei ca. 2.6 liegt, ist eine Schätzung für den Verschleißgrad $\gamma$. Der niedrige Wert lässt einen unbeschädigten Radreifen vermuten. Auch wenn in der Praxis natürlich keine echte Kammfunktion ist, lässt Abb. 1b 
zumindest deutlich erkennen, dass es sich um eine stationäre Funktion handelt, die außerhalb einzelner Schadstellen um 0 herum schwankt.

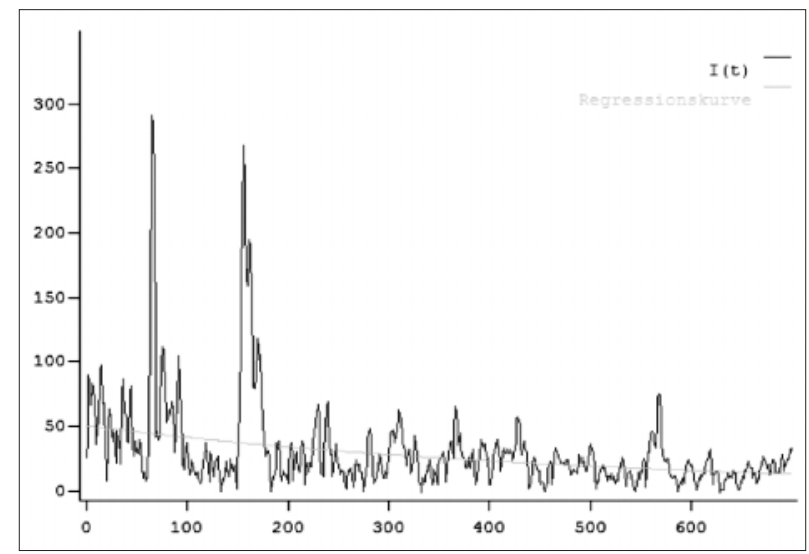

Abbildung $2 a$

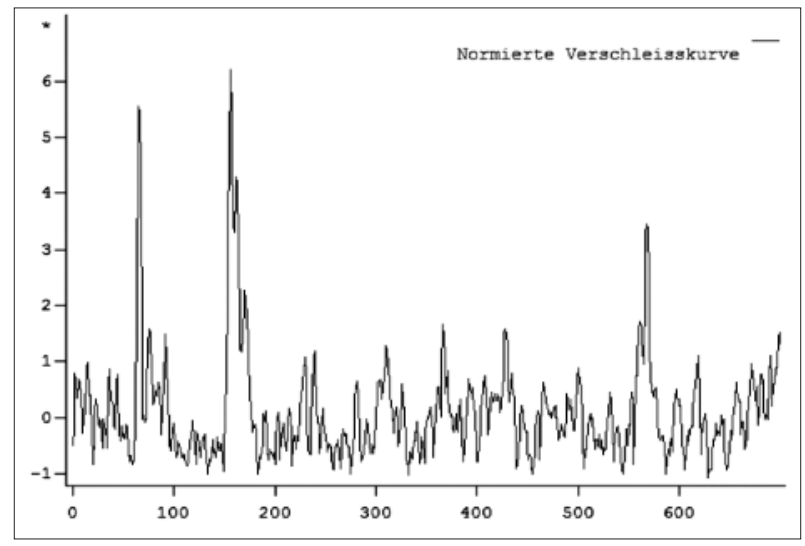

Abbildung $2 b$

Abbildung 2a und 2b zeigen eine entsprechende Auswertung eines bestimmten Radreifens auf der rechten Seite im Datensatz 2004-05-13-20-00-31. Die Maxima der normierten Verschleißkurve liegen bei 5.5 und 6.3 und deuten auf zwei Flachstellen im Radreifen hin. Die Stationarität der Verschleißkurve mit einigen kammartigen Ausbrüchen ist auch hier gut zu erkennen und bestätigt die theoretischen Grundlagen.

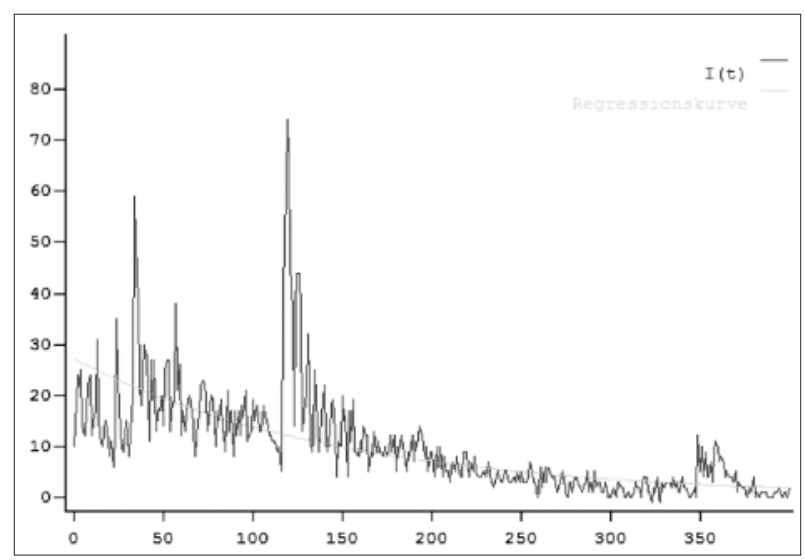

Abbildung $3 a$

Die Analyse des sechsten Radreifens auf der rechten Seite im Datensatz 2004-02-25-19-34-43, der auch in [1] ausführlich betrachtet wurde und als schadhaft bekannt ist, ist in Abbildung 3a und 3b zu sehen. Die Peaks in der Verschleiß- kurve, die teilweise den Wert 5 überschreiten, bestätigen das Vorhandensein einer ausgeprägten Flachstelle.

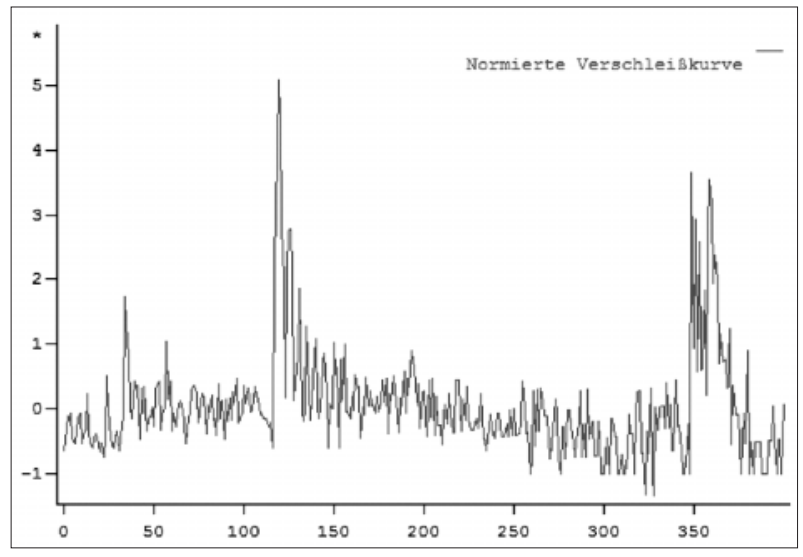

Abbildung $3 b$

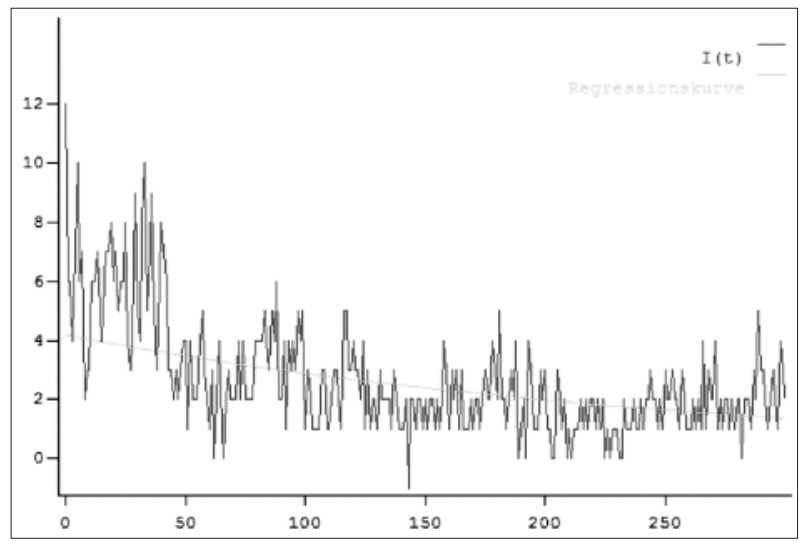

Abbildung $4 a$

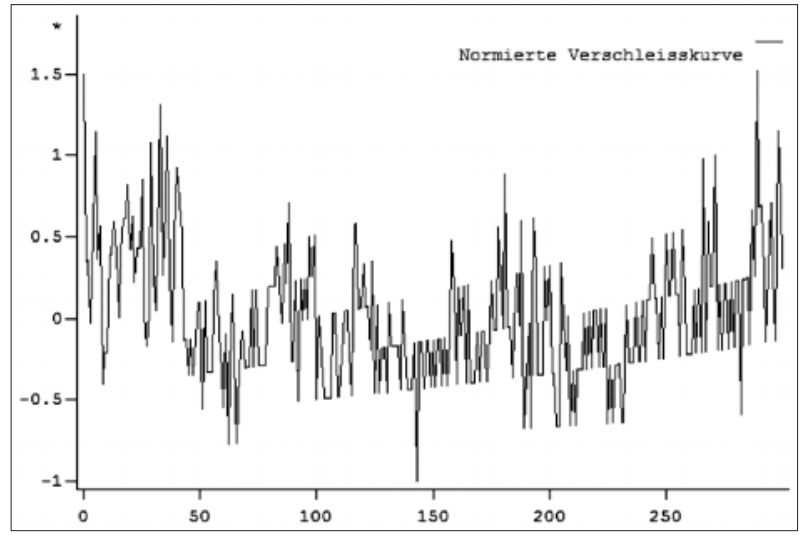

Abbildung $4 b$

Abbildung 4a und 4b zeigen zum Vergleich die Diagramme für den vierten Radreifen auf derselben Seite bei derselben Messung. Niedrige $\gamma$-Werte (durchweg unter 1.5) weisen hier auf einen einwandfreien Radreifen hin.

Der sechste Radreifen auf der linken Seite, der sich auf derselben Achse wie der Radreifen aus Abbildung 3a und $3 \mathrm{~b}$ befindet, weist hingegen deutlich höhere Maxima (ca. 3.8) auf, wie in Abbildung 5a und 5b zu sehen ist. Ein genauer Vergleich der Abbildungen $3 \mathrm{~b}$ und $5 \mathrm{~b}$ zeigt jedoch, dass die zeitliche Position des Maximums in Abb. 5b genau mit der des Maximums in 3b zusammenfällt. Dies deutet klar darauf hin, dass der intensive Schall dieses Impulses über die Achse auf die linke Seite übertragen wurde, der linke Radreifen jedoch selbst nicht beschädigt ist. Beim Auftreten von Flachstellen sind also 
stets die Peaks auf der linken mit denen auf der rechten Seite zu vergleichen, um entsprechende Fehlschlüsse zu vermeiden.

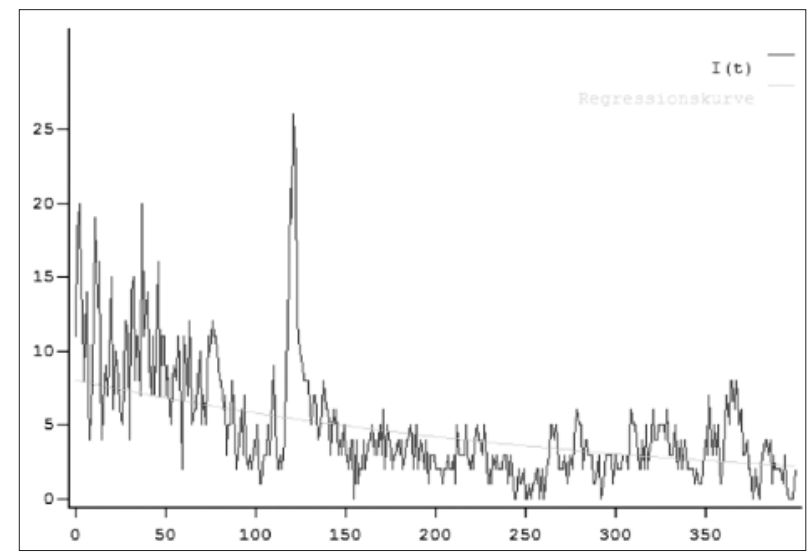

Abbildung $5 a$

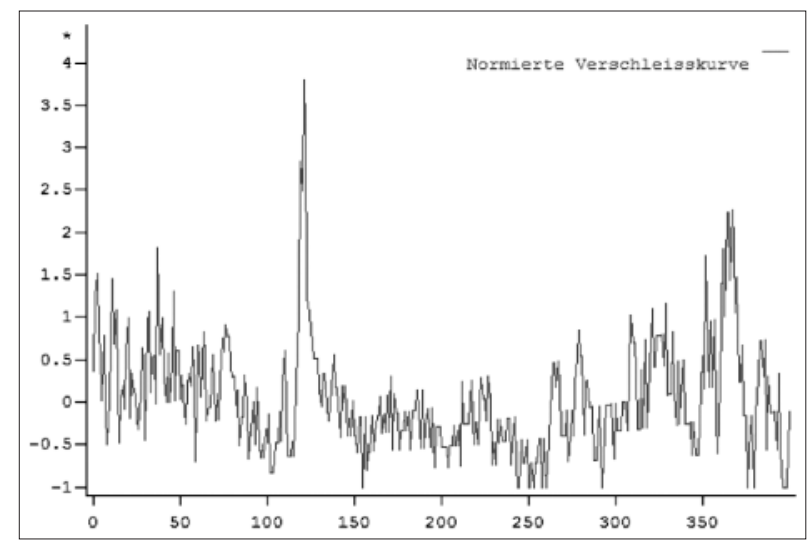

Abbildung $5 b$

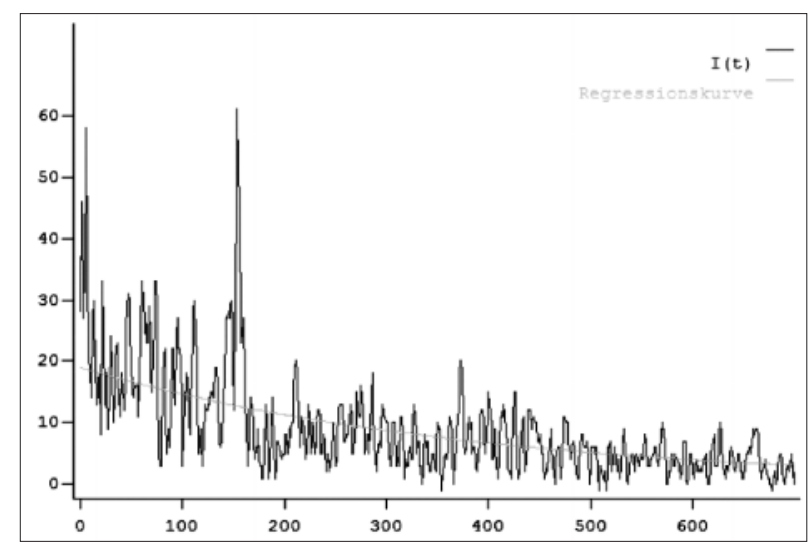

Abbildung $6 a$

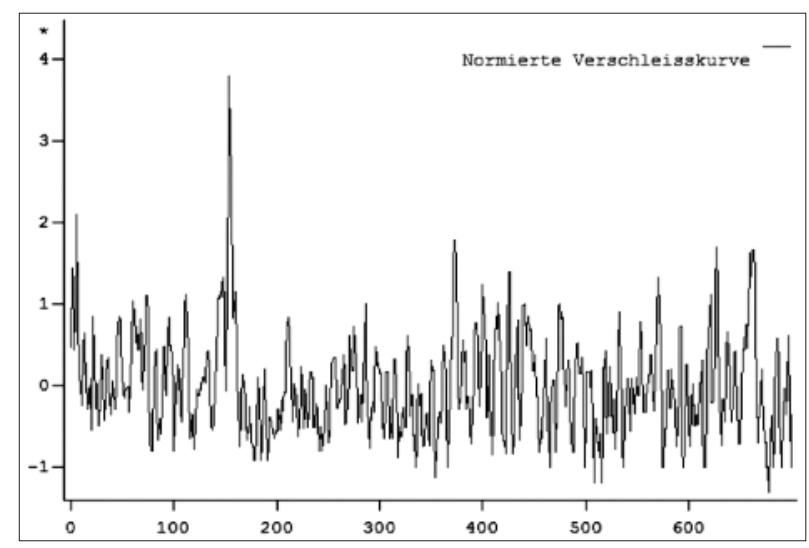

Abbildung $6 b$
Dies bestätigen auch die Untersuchungen am gegenüberliegenden Radreifen zu Abbildung $2 \mathrm{a}$ und 2b, die in Abbildung $6 \mathrm{a}$ und $6 \mathrm{~b}$ zu sehen sind. Das Maximum in Abb. 6b fällt mit einem der Peaks in Abb. 2b zusammen. Da ansonsten die $\Gamma(t)$-Werte niedrig sind, dürfte der Radreifen auf der linken Seite unbeschädigt sein.

\section{Mögliche Probleme}

\subsection{Schätzung des Offsets}

Die Bestimmung des Offsets $I_{R}$ gemäß Abschnitt 3.1 als Maß für den Rauschpegel ist an sich nicht sehr schwierig. Allerdings wirken sich kleine Fehler stark auf das Ergebnis, vor allem auf die normierte Verschleißkurve aus, wie in Abbildung $7 \mathrm{zu}$ sehen ist.

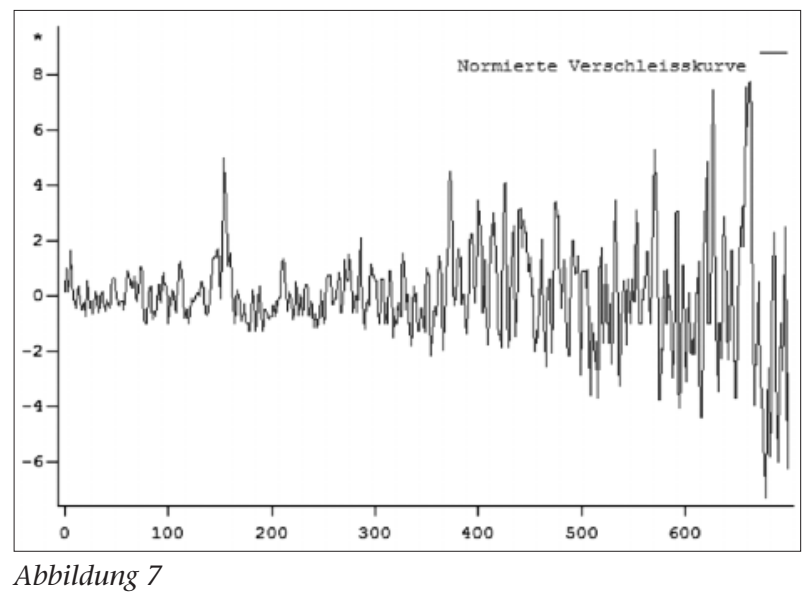

Dort wurde mit $I_{R}=6$ operiert, anstelle des korrekten Wertes von $I_{R}=3$ wie in Abbildung 6b. Es ist offensichtlich, dass die Interpretation eines solchen Ergebnisses zu dem Fehlschluss führen kann, es handele sich um einen schadhaften Radreifen. An der Kurvenform ist der Schätzfehler jedoch meist gut zu erkennen, da die Verschleißkurve dann nicht mehr das von der Theorie erwartete stationäre Verhalten zeigt [2]. Vielmehr schaukelt sie sich entlang der Zeitachse, d.h. mit abklingender Intensität, immer mehr auf.

Aus diesem Grunde sollte nicht nur die Offset-Schätzung sorgfältig vorgenommen werden, sondern auch das betrachtete Zeitintervall für die Regression nicht zu groß gewählt werden. Es sollte voll im Bereich der noch nicht abgeklungenen Intensität liegen, da für niedrige Intensitäten der relative Fehler immer mehr zunimmt. Außerdem erhalten niedrige Intensitätswerte durch die logarithmische Transformation bei der Regression ein überproportionales Gewicht.

\subsection{Güte der linearen Regression}

Wenn die normale Schallintensität eines Radreifens nur durch einzelne Impulse von Flachstellen überlagert wird, ist die Bestimmung der exponentiellen Abklingkurve mit Hilfe der in Abschnitt 3 beschriebenen logarithmisch-linearen Regression von der Theorie gerechtfertigt. Andernfalls, also bei größeren Störungen (z.B. bei über dem gesamten Radreifen verteilten Schadstellen oder Polygonen), ist die Regression zwar auch berechenbar und liefert stets ein eindeutiges Ergebnis, nur ist dieses dann wenig aussage- 
kräftig. Es wird also ein Gütekriterium benötigt, das die automatische Erkennung einer solchen Situation erlaubt. Ein geeignetes Maß für die Güte einer linearen Regression ist der Korrelationskoeffizient $r$, der mit den Bezeichnungen aus Abschnitt 3 definiert ist als

$$
r=\frac{N \sum t_{i} z_{i}-\sum t_{i} \sum z_{i}}{\sqrt{\left(N \sum t_{i}^{2}-\left(\sum t_{i}\right)^{2}\right)\left(N \sum z_{i}^{2}-\left(\sum z_{i}\right)^{2}\right)}}
$$

Der Korrelationskoeffizient liegt aufgrund seiner Definition stets zwischen -1 und 1. In unserem Fall muss er stets negativ sein, da wir eine abklingende Intensität untersuchen. Werte für $r$, die zwischen -1 und -0.5 liegen, deuten auf ein gutes Regressionsergebnis hin. Auch mit Werten um -0.3 wurden bei der Untersuchung der zur Verfügung stehenden Beispieldaten teilweise noch brauchbare Ergebnisse erzielt. Liegt der Korrelationskoeffizient jedoch darüber oder wird sogar positiv, darf mit dem Regressionsergebnis nicht weitergearbeitet werden, da dann das theoretische Modell nicht mehr zu den Daten passt. In diesem Fall kann aber mit hoher Sicherheit von einem (wahrscheinlich stark) beschädigten Radreifen ausgegangen werden, der entweder mehrere Flachstellen oder Polygone besitzt.

\subsection{Störungen durch benachbarte Räder}

Das vorgestellte Verfahren wurde unter der Prämisse entwickelt, dass die Intensitätskurven hintereinander liegender Räder sich nicht überlagern. Tatsächlich ist dies natürlich nicht der Fall. Neben dem nächstgelegenen Radreifen sind in dem gemessenen Schallsignal auch die benachbarten und weiter entfernte Achsen des Schienenfahrzeugs enthalten. Insbesondere die nächstliegende, benachbarte Achse im selben Drehgestell verfälscht das theoretische Modell und somit auch die empirischen Ergebnisse. Um dies zu korrigieren, wäre ein aufwendigeres Modell erforderlich, das dann allerdings nicht mehr mit einer einfachen linearen Regression geschätzt werden kann. Im nächsten Abschnitt wird eine solche Erweiterungsmöglichkeit angesprochen, die in einem Anschlussprojekt realisiert werden könnte.

\section{Fazit und Ausblick}

\subsection{Zusammenfassung}

In diesem Projekt wurde ein Verfahren entwickelt, mit dessen Hilfe aus Körperschallsignalen Flachstellen an Radreifen detektiert werden können. Zunächst wurde ein theoretisches Modell aufgestellt, das den Intensitätsverlauf des Schallsignals und einzelner Störimpulse durch Flachstellen beschreibt. Daraus wurde die Definition einer dimensionslosen Kenngröße abgeleitet, des sog. normierten Verschleißgrades $\gamma$, der ein Maß für die Beschädigung eines Radreifens darstellt. Es konnte gezeigt werden, dass der Verschleißgrad $\gamma$ weitgehend invariant gegen die Kalibrierung des Sensors und die Überfahrtgeschwindigkeit des Schienenfahrzeugs ist. Es wurde ein Algorithmus vorgestellt, mit dem der Verschleißgrad empirisch aus realen Messungen am Schienenkörper ermittelt werden kann. An einigen Beispieldaten wurden die Ergebnisse des Verfahrens illustriert und abschließend auf mögliche Fallstricke, die in der Praxis auftreten können, sowie Möglichkeiten ihrer Abhilfe hingewiesen.

Zum Schluss sollen noch Perspektiven aufgezeigt werden, wie das Verfahren in Zukunft weiter verbessert werden kann.

\subsection{Nicht-lineares Fitting}

Die Anpassung der gesuchten Exponentialkurve mit Hilfe einer logarithmisch-linearen Regression ist aus numerischer Sicht zwar einfach zu handhaben und effizient zu berechnen, sie bringt aber einige Nachteile mit sich. Die zunächst durchgeführte logarithmische Transformation verzerrt nämlich die Messdaten, so dass niedrige Intensitäten ein hohes Gewicht und hohe Intensitäten ein niedriges Gewicht erhalten. Dies hat zur Folge, dass die Regressionskurve nicht die Exponentialkurve mit der geringsten mittleren quadratischen Abweichung von der Messkurve ist. Außerdem können (durch Schwankungen bedingte) negative Werte nicht berücksichtigt werden, da der Logarithmus negativer Zahlen nicht reellwertig ist.

Will man die Kurve mit der geringsten quadratischen Abweichung ermitteln, muss man auf nicht-lineare Mehrschritt-Verfahren zurückgreifen. Mit Hilfe des nicht-linearen, iterativen Optimierungsverfahrens von Marquardt und Levenberg [4] kann die Exponentialfunktion mit der geringsten quadratischen Abweichung von der gemessenen Intensität direkt bestimmt werden (sog. Fitting), ohne dass die Daten vorher geglättet oder transformiert werden müssen. Ein weiterer Vorteil dieses Verfahrens ist, dass der Offset $I_{R}$ nicht separat bestimmt werden muss, sondern beim Fitting als Abfallprodukt mitberechnet werden kann. Diese Vorteile werden allerdings mit der Tatsache erkauft, dass der Algorithmus komplizierter und rechenaufwendiger ist als Regressionsverfahren.

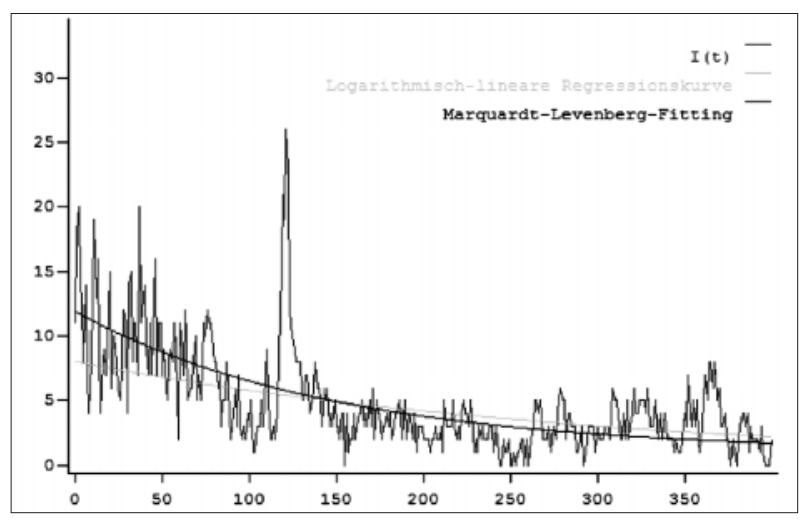

Abbildung 8

Abbildung 8 zeigt den Vergleich der beiden Verfahren am Beispiel des linken Rades auf der sechsten Achse in der Potsdamer Messung (vgl. Abb. 5a). Erwartungsgemäß passt sich das nicht-lineare Fitting besser an die hohen Intensitätswerte an als die logarithmisch-lineare Regression.

$\mathrm{Zu}$ beachten ist, dass bei Verwendung des FittingVerfahrens anstelle der Regression der Grenzwert des Verschleißgrades $\gamma$, ab dem von einem schadhaften Radreifen ausgegangen werden muss, $u$. U. anders geeicht werden muss, da sich die Fittingkurve, durch die bei der Berechnung von $\Gamma(t)$ dividiert werden muss, auf einem anderen Niveau befindet. 


\subsection{Multifunktionaler Ansatz}

Wie bereits in Abschnitt 6 angesprochen, müssten für eine genauere Modellierung die Beiträge aller $R$ Räder auf einer Schiene berücksichtigt werden. Die Intensitätsfunktion hätte dann anstelle von (9) die Form:

$$
I(t)=I_{R}+\sum_{m=1}^{R} f_{\lambda v_{m}}\left(t-t_{0, m}\right)\left[\alpha_{m}+\beta_{m} \sum_{k=-\infty}^{\infty} \delta\left(t-k \Delta t-t_{d, m}\right)\right]
$$

Diese komplexe Funktion kann jedoch leider weder durch Regression noch durch nicht-lineares Fitting stabil geschätzt werden. Die gesuchten Parameter müssen deshalb in mehreren Schritten bestimmt werden. Die Überfahrtgeschwindigkeiten $v_{m}$ können z.B. mit dem Induktionssensor direkt gemessen werden. Die Dämpfungskonstante $\lambda$ ist eine (konstante) Eigenschaft der Schiene und ihrer Einfassung. Sie könnte anhand von einigen Referenzmessungen hinreichend genau mit Hilfe der Marquardt-LevenbergMethode aus Abschnitt 7.2 bestimmt werden. Für die verbleibenden Parameter müsste ein geeignetes, multilineares Regressionsverfahren zum Einsatz kommen, das eine schnelle, genaue und robuste Schätzung dieser Parameter erlaubt. Mit Hilfe einer entsprechend erweiterten Theorie sollte daraus dann der Verschleißgrad $\gamma$ sehr zuverlässig ermittelt werden können.

\section{Literatur}

[1] Manfred Deutzer: DTK-System zur Ermittlung von Flachstellen bei einer Fahrzeugüberfahrt. Fachbeitrag DTK, 2004.

[2] Ralf Vandenhouten: Analyse instationärer Zeitreihen komplexer Systeme. Shaker Verlag, 1998.

[3] A. V. Oppenheim, R. W. Schafer: Discrete-Time Signal Processing. Prentice Hall, 1989.

[4] William H. Press et al.: Numerical Recipes. Cambridge University Press, 1993

[5] Die Software Dataplore ${ }^{\circledR}$ (http://dataplore.com) zur Zeitreihen- und Signalanalyse wurde in diesem Projekt für die Entwicklung und Verifikation des beschriebenen Verfahrens verwendet.

\section{Autoren}

Prof. Dr. rer. nat. Ralf Vandenhouten

Technische Fachhochschule Wildau

Fachbereich Ingenieurwesen/Wirtschaftsingenieurwesen Lehrstuhl für Telematik

Tel. +49 3375 508-359

rvandenh@tfh-wildau.de

Dipl.-Chemiker Manfred Deutzer

Deutzer Technische Kohle GmbH

Lindenallee 16, 15738 Zeuthen

Tel. +4933762 21292

info@deutzer.de 